\title{
BMJ Open Impact of incremental versus conventional initiation of haemodialysis on residual kidney function: study protocol for a multicentre feasibility randomised controlled trial
}

\author{
Raja Mohammed Kaja Kamal (D) ,,2 Ken Farrington,, ${ }^{1,2}$ David Wellsted, ${ }^{2}$ \\ Sivakumar Sridharan, ${ }^{1,2}$ Bassam Alchi, ${ }^{3}$ James Burton, ${ }^{4}$ Andrew Davenport, ${ }^{5}$ \\ Enric Vilar ${ }^{1,2}$
}

To cite: Kaja Kamal RM, Farrington $\mathrm{K}$, Wellsted $\mathrm{D}$, et al. Impact of incremental versus conventional initiation of haemodialysis on residual kidney function: study protocol for a multicentre feasibility randomised controlled trial. BMJ Open 2020;10:e035919. doi:10.1136/ bmjopen-2019-035919

- Prepublication history and additional material for this paper are available online. To view these files, please visit the journal online (http://dx.doi. org/10.1136/bmjopen-2019035919).

Received 21 November 2019 Revised 26 April 2020 Accepted 29 May 2020

Check for updates

(c) Author(s) (or their employer(s)) 2020. Re-use permitted under CC BY-NC. No commercial re-use. See rights and permissions. Published by BMJ.

For numbered affiliations see end of article.

Correspondence to

Dr Enric Vilar;

enric.vilar@nhs.net

\section{ABSTRACT}

Introduction Preserving residual kidney function (RKF) may be beneficial to patients on haemodialysis (HD) and it has been proposed that commencing dialysis incrementally rather than three times a week may preserve RKF. In Incremental HD, target dose includes a contribution from RKF, which is added to HD dose, allowing individualisation of the HD prescription. We will conduct a feasibility randomised controlled trial (RCT) comparing incremental HD and conventional three times weekly treatments in incident HD patients. The study is designed also to provide pilot data to allow determination of effect size to power a definitive study.

Methods and analysis After screening to ensure native renal urea clearance $>3 \mathrm{~mL} / \mathrm{min} / 1.73 \mathrm{~m}^{2}$, the study will randomise 54 patients within 3 months of $\mathrm{HD}$ initiation to conventional in-centre thrice weekly dialysis or incremental in-centre HD commencing 2 days a week. Subjects will be followed up for 12 months. The study will be carried out across four UK renal centres.

The primary outcome is to evaluate the feasibility of conducting a definitive RCT and to estimate the difference in rate of decline of RKF between the two groups at 6 and 12 months time points. Secondary outcomes will include the impact of dialysis intensity on vascular access events, major adverse cardiac events and survival. Impact of dialysis intensity on patientreported outcomes measures, cognition and frailty will be assessed using EQ-5D-5L, PHQ-9, Illness Intrusiveness Rating Score, Montreal Cognitive assessment and Clinical Frailty Score. Safety outcomes include hospitalisation, fluid overload episodes, hyperkalaemia events and vascular access events.

This study will inform the design of a definitive study, adequately powered to determine whether RKF is better preserved after incremental HD initiation compared with conventional initiation.

Ethics and dissemination Ethics approval has been granted by Cambridge South Research Ethics Committee, United Kingdom(REC17/EE/0311). Results will be disseminated via peer-reviewed publication.

Trial registration number NCT03418181
Strengths and limitations of this study

- There are no randomised studies comparing incremental haemodialysis (HD) and conventional three times weekly treatments in incident HD patients. This study will address this gap.

- It will provide data on feasibility of recruitment to a definitive study together with an estimate of the effect size of group differences in rate of loss residual kidney function (RKF) allowing sample size calculation.

- Impact and intrusiveness of dialysis intensity will also be compared between groups.

- The sample size will not permit definitive determination of differences in the rate of decline of RKF between groups.

\section{BACKGROUND}

Most patients with end-stage renal failure have a degree of native kidney function (residual kidney function, RKF) remaining when they initiate haemodialysis (HD). There has been recent interest in incremental $\mathrm{HD}$, a method of individualising HD according to the level of RKF to permit dialysis to be commenced at a lower intensity than conventional approaches allow. Most patients commence dialysis using conventional three times weekly dialysis with RKF usually not accounted for in prescribing dialysis dose. In Incremental $\mathrm{HD}, \mathrm{RKF}$ is combined with dialysis clearance to provide an overall measure of solute removal allowing the dose provided by dialysis to be individualised. Various algorithms are available to assist with this such as Standard Kt/V (Std $\mathrm{Kt} / \mathrm{V}$ ), which includes contributions from both Std Kt/ $\mathrm{V}_{\mathrm{RKF}}$ and $\mathrm{Std} \mathrm{Kt} / \mathrm{V}_{\text {dialvsis }}{ }^{1-3}$ In this approach, reduction of dialysis dose may be considered provided that the combined urea 
clearance targets are met and other markers of dialysis adequacy such as blood pressure, interdialytic weight gains, anaemia, potassium, phosphate control, nutrition and general well-being are not compromised. The technique requires that the proportion of target dose provided by dialysis is increased as the RKF declines or if there are any other indicators for inadequate dialysis. The dialysis team and patients need to be aware of the importance of regular measurement of RKF 1-3 monthly. ${ }^{4}$ This incremental approach may not be suitable for patients who are unable or unwilling to collect urine samples.

Traditionally RKF has been incorporated into peritoneal dialysis dosing but it has not been included in calculating HD dose due to limited practical experiences and outcome data from clinical studies. There are no randomised controlled trials (RCTs) that compare clinical outcomes of incremental HD and those of conventional thrice-weekly HD. A number of observational studies have compared clinical outcomes of twice-weekly HD and conventional thrice-weekly HD regimens. ${ }^{5-19}$ These studies suggest that the mortality risks and survival outcomes are not inferior in those on the twice weekly dialysis regimen compared and those treated conventionally, provided there is adequate RKF. Importantly, several non-randomised studies have suggested that RKF is better preserved in those dialysed twice weekly commencing soon after dialysis initiation. ${ }^{6} 10111718$ Preservation of RKF may provide clinical benefits to HD patients including better fluid control, significant solute and fluid removal. It is also associated with improved quality of life and survival.

These findings indicate the need for a prospective RCT comparing RKF preservation following incremental and conventional initiation of dialysis. We are undertaking a study to determine the feasibility of conducting such a study. Our study will also provide pilot data to estimate differences in the rate of decline of RKF in the first year after commencing dialysis using either conventional or incremental approaches. The primary outcome of our study is to evaluate the feasibility of conducting an RCT in patients who have recently started HD. Patients will be randomised either to an incremental arm initiating with twice weekly dialysis or to a conventional three times weekly dialysis. Our study will explore key methodological, design and safety issues, and also estimate an effect size. These findings will facilitate the design of a subsequent definitive study.

\section{METHODS/DESIGN}

\section{Funding and governance}

The trial is sponsored by East and North Hertfordshire National Health Service (NHS) Trust. The University of Hertfordshire Clinical Trial Support Network (CTSN) will provide independent support for randomisation and monitoring of the study. The conduct of the trial will be overseen by a Steering group which will meet regularly and will include an independent chair and coapplicants.
The CTSN will monitor compliance with the study protocol at 3 months following study initiation and then as required by sponsor scrutiny of data returns.

\section{Ethics and dissemination}

The study received ethical approval from East of EnglandCambridge South (REC reference 17/EE/0311; IRAS project ID 219032). Study endpoints, whether negative or positive, will be published with the intention of reaching a wide audience in nephrology both in peer-reviewed publication and also submitted for presentation at international and UK meetings including the British Renal Society Conference. Following publication of final data an anonymised data set will be made available on request.

\section{Patient and public involvement}

A summary of the initial protocol was shared with 10 patients who were asked to comment on the study design, the potential willingness of patients to participate in the study and the burden of study procedures and interventions. Their comments were taken account of in preparing the final version of the protocol. Patients will be involved in interpreting study finding and in design of definitive study. We will report a summary of results to patients in a personal communication by mail. We will also summarise results to local patient association newsletters.

\section{Setting}

The study will take place in four NHS Trust renal unitsEast and North Hertfordshire, Royal Free Hospitals, Royal Berkshire Hospitals and University Hospitals of Leicester. The total number of participants from all centres will be 54. Recruitment commenced in January 2018 and completion of follow-up will be in May 2020.

\section{Study objectives and end points}

The study's primary objective is to determine the feasibility of conducting a definitive RCT of incremental HD initiation, compared with conventional thrice weekly in-centre HD initiation. There are a number of aspects to this primary objective which are summarised in table 1 . We will determine, at each study site, the proportion of incident HD patients it is practical to approach, who prescreen as suitable for formal study screening (eligibility for screening). We will determine the proportion of those patients who consent undergo formal screening, pass the screening test and are randomised (recruitability). We will also determine the study retention rate (retainability) as well as fidelity to the protocol (protocol adherence) of patients in the study. Numerators and denominators for these parameters are shown in figure 1. The study will establish evidence for the safety of the incremental approach. It will also generate data allowing estimation of the effect size of the difference in rate of decline of RKF in the 6 months following randomisation between incremental and conventional HD arms.

Secondary objectives of the study are to determine whether there is a signal of benefit for incremental HD initiation for improving Quality of Life, mood, cognitive 
Table 1 Study objectives

\begin{tabular}{|c|c|}
\hline Primary objective & Primary outcome \\
\hline $\begin{array}{l}\text { The proportion of eligible subjects agreeing to participate } \\
\text { in the study-Recruitability }\end{array}$ & $\begin{array}{l}\text { Proportion incident HD patients it is practical to approach, who prescreen as } \\
\text { suitable for screening (eligibility for screening). } \\
\text { Proportion of screened patients who fulfil all eligibility criteria for participation } \\
\text { in the study. } \\
\text { Proportion of these patients who agree to participate in the study. }\end{array}$ \\
\hline $\begin{array}{l}\text { The proportion of randomised subjects who remain in the } \\
\text { study-Retainability }\end{array}$ & $\begin{array}{l}\text { Proportion of patients randomised who remain in the study excluding study } \\
\text { withdrawals, and reasons for withdrawals. }\end{array}$ \\
\hline $\begin{array}{l}\text { The number of adverse and serious adverse events- } \\
\text { Safety of the study }\end{array}$ & $\begin{array}{l}\text { Frequency of hospital admission due to hyperkalaemia and fluid overload, and } \\
\text { lower respiratory tract infection. }\end{array}$ \\
\hline $\begin{array}{l}\text { An estimate of the effectiveness of the intervention- } \\
\text { Effect size }\end{array}$ & $\begin{array}{l}\text { Dialysis dose and RKF as measured by Std Kt/N. } \\
\text { Rate of change (mean) of RKF in the first } 6 \text { and } 12 \text { months after randomisation. }\end{array}$ \\
\hline Mood-depression & Depression assessed using PHQ-9 questionnaire. \\
\hline Cognitive function & Change in cognitive function as assessed by MOCA tool. \\
\hline Illness intrusiveness & IIIness intrusiveness is assessed using IIIness Intrusiveness Rating Scale. \\
\hline Functional status/frailty & Functional status assessed by Clinical Frailty Score. \\
\hline Vascular access failures or problems & Frequency of vascular access failures and interventions. \\
\hline Major adverse cardiac events (MACE) & MACE is assessed by recording of the frequency of the events. \\
\hline Survival & Survival is measured by all-cause mortality. \\
\hline
\end{tabular}

EQ-5D-5L, EuroQol EQ-5D-5L questionnaire; HD, haemodialysis; MOCA, Montreal Cognitive assessment; PHQ-9, Patient Health Questionnaire, 9 question; RKF, residual kidney function; Std Kt/N, Standard Kt/N.

function, illness intrusiveness, functional status, frailty, risk of vascular access failure or interventions, major adverse cardiac events (MACEs) and survival. Specific tools used and methods to measure secondary outcomes related to these secondary objectives are detailed in table 1. Illness intrusiveness will be measured with the Illness Intrusiveness Rating Scale (IIRS), a validated tool to measure impact of the dialysis treatment and disease on physiologically meaningful activity and its psychosocial impact. ${ }^{20}$ Quality of life will be measured using EQ-5D-5L, a validated tool which will capture different dimensions of quality of life including anxiety/depression and pain/ discomfort and can be used in health economic evaluation. ${ }^{21}$ Cognitive function will be measured using the Montreal Cognitive Assessment (MoCA) which is a tool for assessment of cognitive function that has been validated in dialysis patients against detailed neurophysiological testing covering different domains of cognitive function and provides good sensitivity and specificity for identifying cognitive impairment in this population. ${ }^{22} 23$ Clinical frailty will be measured using the Clinical Frailty Score (CFS). ${ }^{24} 25$

\section{Participants}

All adult patients who have commenced HD in the previous 3 months will be considered for the study. Those who potentially meet the eligibility criteria after prescreening by review of medical records including the requirement for a standard of care interdialytic urea clearance $\geq 3 \mathrm{~mL} / \mathrm{min} / 1.73 \mathrm{~m}^{2}$ Body Surface Area (BSA) will eligible for study screening. Those consenting for the study will undergo formal screening to include confirmation of their meeting the eligibility criteria including having an interdialytic urea clearance $\geq 3 \mathrm{~mL} / \mathrm{min} / 1.73$ $\mathrm{m}^{2} \mathrm{BSA}$ on retesting.

\section{Consent}

Consent will be required prior to screening procedures and will include agreement to screening which includes confirmation of urea clearance $\geq 3 \mathrm{~mL} / \mathrm{min} / 1.73 \mathrm{~m}^{2}$ BSA and an explicit consent to a protocol-driven dialysis regimen and to randomisation to incremental HD or standard thrice weekly HD arms.

Inclusion criteria:

- Age $\geq 18$ years.

- Advanced kidney failure-established as a new starter on HD within the previous 3 months.

- RKF likely to permit twice weekly dialysis as defined by interdialytic urea clearance $\geq 3 \mathrm{~mL} / \mathrm{min} / 1.73 \mathrm{~m}^{2}$ BSA measured routinely as part of standard care or as prescreening.

- Sufficient understanding of the study procedures and requirements including capacity for explicit agreement to be randomised to standard or incremental HD regimens. 


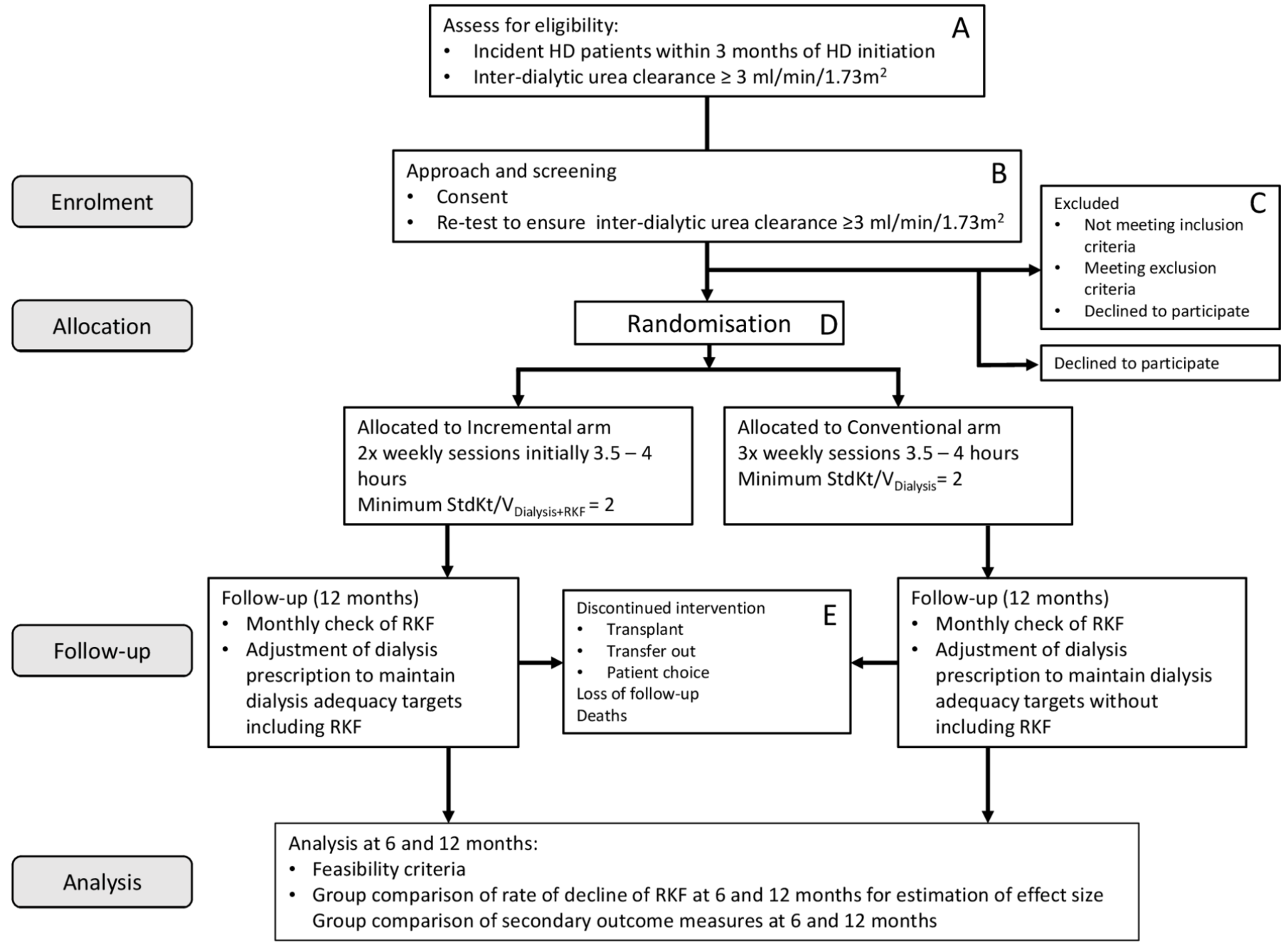

\section{Eligibility: A Recruitability: D/(B-C) Drop-out rate: $\mathrm{E}$}

Figure 1 Flow diagram of clinical trial demonstrating data that will be used to calculate eligibility for screening, screen failure rate, recruitability and retainability. HD, haemodialysis; RKF, residual kidney function; Std Kt/N, Standard Kt/V.

Exclusion criteria

- Planned organ transplantation within 3 months from study screening.

- Anticipated requirement for high-volume ultrafiltration on dialysis (eg, subjects with daily enteral or parenteral nutrition)

- Bloodborne virus positivity.

- Subjects unable to comply with requirement for monthly interdialytic urine collection.

- Pregnancy.

- Prognosis $<12$ months as judged by the principal investigator.

\section{Screening phase}

At screening inclusion and exclusion criteria will be confirmed. Confirmation of interdialytic urea clearance $\geq 3 \mathrm{~mL} / \mathrm{min} / 1.73 \mathrm{~m}^{2}$ BSA will be performed. Pregnancy test will be performed in females of childbearing age to reduce chance of unexpected pregnancy occurring during the study which would require study withdrawal. Patients who, at screening, are eligible for study participation according to eligibility criteria, and who are confirmed to have a screening inter-dialytic urea clearance $\geq 3 \mathrm{~mL} / \mathrm{min} / 1.73 \mathrm{~m}^{2}$ BSA will eligible for randomisation. Subjects who fail screening will be eligible for rescreening 1 month later provided their screening urea clearance is $>2 \mathrm{~mL} / \mathrm{min} / 1.73 \mathrm{~m}^{2} \mathrm{BSA}$ and the rescreening time point remains within 3 months of dialysis initiation. At rescreening, a urea clearance $\geq 3 \mathrm{~mL} / \mathrm{min} / 1.73 \mathrm{~m}^{2}$ BSA will be required for randomisation into the study.

\section{Randomisation}

Web-based randomisation will be carried out by each centre using Qualtrics, supported by the CTSN, University of Hertfordshire. Subjects will be randomised on a 1:1 basis to each study arm and each subject allocated a unique study ID. 


\section{Study phase}

Following randomisation, study subjects will be dialysed according to the protocol of their randomisation arm as per the schematic in figure 1. Monthly quality assessment of dialysis in both arms will include a measure of dialysis clearance ( $\mathrm{Std} \mathrm{Kt} / \mathrm{V}_{\text {Dialysis }}$ ). RKF will be measured monthly by urea clearance in both arms and converted to Std Kt/ $\mathrm{V}_{\text {RKF}}$.

In the standard dialysis arm, dialysis adequacy will be assessed only using the $\mathrm{Std} \mathrm{Kt} / \mathrm{V}_{\text {Dialysis }}$. In the incremental dialysis arm, the adequacy will be assessed using a composite of dialysis clearance ( Std Kt $\left./ \mathrm{V}_{\text {Dialysis }}\right)$ and $\operatorname{RKF}\left(\mathrm{Std} \mathrm{Kt} / \mathrm{V}_{\mathrm{RKF}}\right.$ ) as detailed below. This composite is termed Std Kt $/ \mathrm{V}_{\text {Dialysis+RKF }}$. HD modes will remain standard throughout the study. Haemodiafiltration may be used where blood flow $>250 \mathrm{~mL} / \mathrm{min}$, otherwise high-flux HD will be used.

\section{Assessment of RKF}

There are two main methods of including RKF in HD prescription. The first converts residual urea clearance to an equivalent dialysis sessional clearance. ${ }^{7}$ The second converts sessional $\mathrm{Kt} / \mathrm{V}$ to a weekly equivalent clearance. Both these allow the addition of dialysis and renal clearances. There are two variants of the second method: Std $\mathrm{Kt} / \mathrm{V}^{26}$ and the Casino-Lopez Equivalent renal urea clearance (EKR) ${ }^{27}$ Both these are urea clearance based. The ERBP guidelines recommend use of GFR (mean of urea and creatinine clearance) in the EKR equation rather than urea clearance which was intrinsic to originally derived equation. We have used Std Kt/V which takes a more conservative view of RKF since urea clearance is around $30 \%$ lower than GFR. Further details of the methodology for assessment of RKF can be found in online supplementary material 1.

\section{Groups}

Control Group: standard HD arm

Subjects in the standard HD arm will be dialysed to target minimum Std $\mathrm{Kt} / \mathrm{V}_{\text {Dialysis }}$ of 2 per week. Subjects will be dialysed after randomisation initially for 3.5-4 hours thrice weekly. Dialysis dose will be adjusted using standard measures including maximising blood flow, dialysis time, membrane surface area and improving vascular access. Reduction in dialysis frequency will not be permitted.

\section{Interventional group: incremental HD arm}

Subjects randomised to the incremental HD arm will be dialysed to a target minimum Std Kt/ $\mathrm{V}_{\text {Total }}$ (Std Kt/ $\mathrm{V}_{\text {dialysis }}+\mathrm{Std} \mathrm{Kt} / \mathrm{V}_{\mathrm{RKF}}$ ) of 2 per week. Following randomisation dialysis will be initiated twice weekly, with a session duration of 3.5-4 hours. If Std $\mathrm{Kt} / \mathrm{V}_{\text {Total }}$ exceeds the minimum target, clinicians will be permitted to reduce dialysis duration provided the target level is still achieved. If $\mathrm{Std} \mathrm{Kt} / \mathrm{V}_{\text {Total }}$ does not meet the target, clinicians will be permitted to increase dialysis dose by optimising dialysis clearance (membrane selection, blood flow, vascular access, increasing dialysis time or frequency). Clinicians will also be permitted to increase the dialysis frequency to thrice-weekly or greater if required. The main trigger for this will be failure to meet minimum adequacy targets but clinicians will have the freedom to make this transition on other clinical grounds including hyperkalaemia and fluid overload. The reasons for switching from twice to thrice weekly will be recorded. Hyperkalaemia and fluid overload are also captured as serious adverse events (SAEs).

\section{Deviations to study protocol}

If subjects are admitted to hospital, efforts will be made to maintain adherence to the dialysis protocol. However, during admissions, modifications to the dialysis prescription, which include increasing dialysis frequency, are permitted in the interests of patient safety. These will be recorded as protocol deviations.

In the event of subjects in the incremental HD arm not providing interdialytic urine samples for calculation of Std $\mathrm{Kt} / \mathrm{V}_{\text {Renal }}$ for two consecutive months, the subject will be advised to dialyse thrice weekly and will remain in the study with target $\mathrm{Std} \mathrm{Kt} / \mathrm{V}_{\text {Dialysis }}>2$ (ie, assuming $\mathrm{RKF}$ is zero), until an interdialytic urine collection is provided. Additional study visits may be performed if necessary following hospital admission, holiday or non-adherence to treatment schedule.

\section{Procedures to avoid loss from follow-up or study withdrawal}

The patient information sheet (online supplementary material 2) and consent form (online supplementary material 3)will draw attention to the requirement for patients to agree that their dialysis regimen and frequency will be adjusted according to the study protocol.

For patients wishing to withdraw consent, the investigator will explore with the patient the reasons for wishing to withdraw. In patients who wish to withdraw because they are unable to tolerate the intensity, frequency or duration of dialysis, the investigator will be permitted to offer to the patient to remain in the study with reduced dialysis intensity according to clinical judgement and record this as a protocol deviation (intention-to-treat approach). Patients who withdraw will be encouraged to remain in the study for the purpose of outcome data collection including measurement of RKF.

\section{Data collection}

Data will be collected by the research team members at baseline and then monthly thereafter for 12 months. Table 2 summarises study assessments during the study and study time points.

\section{Measurement of dialysis adequacy}

Details of the method of measuring dialysis adequacy are provided in online supplementary material 1 . The dialysis dosing adjustment will be carried out monthly using Std Kt/V calculated by this method. For patients dialysing thrice weekly (Monday/Wednesday/Friday or Tuesday/ Thursday/Saturday), the Monday/Tuesday session is considered to be session 1 of the week (HD1) and the Wednesday/Thursday session is considered session 2 
Table 2 Schedule of events

\begin{tabular}{|c|c|c|c|}
\hline & Study period & & \\
\hline & Prescreening & Baseline/screening & Visit 1-12 \\
\hline Months & -12 to 0 & 0 & $1-12$ \\
\hline Study procedures/assessments & & & \\
\hline Consent & & $\mathbf{x}$ & \\
\hline Inclusion/exclusion criteria & $\mathbf{x}$ & $\mathbf{x}$ & \\
\hline Demographics, medical history, physical examination, height & & $\mathbf{x}$ & \\
\hline Randomisation & & $\mathbf{x}$ & \\
\hline Rescreening $^{*}$ & & $\mathbf{x}$ & \\
\hline $\begin{array}{l}\text { Concomitant medications -diuretics, erythropoietin stimulating } \\
\text { agents, antihypertensive, phosphate binders }\end{array}$ & & $\mathbf{x}$ & $\mathbf{X}$ \\
\hline Monthly dialysis blood tests & & $\mathbf{x}$ & $\mathbf{x}$ \\
\hline Monthly dialysis adequacy assessments & & $\mathbf{x}$ & $\mathbf{X}$ \\
\hline Pre-HD1 urea, post-HD1 urea, pre-HD2 urea, post-HD2 urea† & $\mathbf{x}$ & $\mathbf{x}$ & $\mathbf{x}$ \\
\hline $\begin{array}{l}\text { Inter-dialytic urine collection for urea and creatinine clearance } \\
\text { measurement }\end{array}$ & & $\mathbf{x}$ & $\mathbf{x}$ \\
\hline Frozen samples for $\beta-2$ microglobulin and $\beta$ trace protein & & $\mathbf{x}$ & $\mathbf{x}$ \\
\hline Bioimpedence measurement & & $\mathbf{x}$ & $\mathbf{x}$ \\
\hline Safety assessments & & & \\
\hline Adverse events, serious adverse events, MACE, end points & & & $\mathbf{X}$ \\
\hline Questionnaires & & & \\
\hline EQ-5D-5L, IIRS, PHQ9, MoCA, CFS & & Months 0, 6, 12 & \\
\hline
\end{tabular}

*Patients who fail screening will be eligible for rescreening 1 month later provided their screening urea clearance is $>2 \mathrm{~mL} / \mathrm{min} / 1.73 \mathrm{~m}{ }^{2} \mathrm{BSA}$ and the rescreening time point remains within 3 months of HD initiation.

†Dialysis adequacy can be calculated using either post-HD1 urea, pre-HD2 urea, post-HD2 urea or optionally using pre-HD1 urea, post-HD1 urea, pre-HD2 urea.

CFS, Clinical Frailty Score; HD, haemodialysis; IIRS, Illness intrusiveness rating score; MACE, major adverse cardiac events; MOCA, Montreal Cognitive assessment.

of the week (HD2). For patients dialysing twice weekly (Monday/Friday or Tuesday/Saturday), the Friday/ Saturday is considered HD1 and the Monday/Tuesday HD2. Blood and urine samples to be taken are shown in table 2 (Schedule of Events) and in figure 2. The urine collection and measurement of RKF is performed from HD1 to HD2 and will be calculated from post-HD1 and pre-HD2 serum urea/creatinine, urine volume and urine urea/creatinine concentration as per the equations in

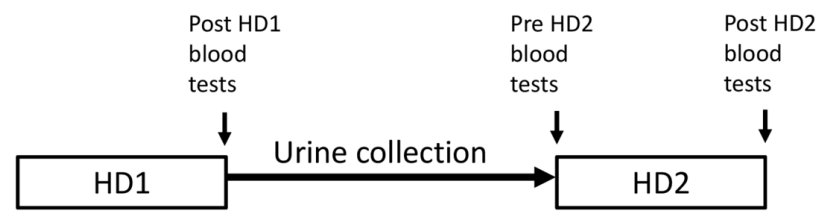

Mon for Mon/Wed/Fri dialysis Tue for Tue/Thurs/Sat dialysis

Fri for Mon/Fri dialysis Sat for Tue/Sat dialysis

Figure 2 Timing of urine collection and blood tests for dialysis adequacy measurement for patients on twice Weekly and thrice weekly HD. HD, haemodialysis. the online supplementary material 1 . The measurement of dialysis dose is calculated from dialysis session data (preweight and postweight HD2 weight, Watson volume, pre-HD2 and post-HD2 urea and dialysis session duration (Td) (see online supplementary material 1 for calculation procedure).

Urine collection will consequently be over approximately 3 days for twice weekly patients and 2 days for thrice weekly patients. Although there is a small risk of bias due to longer duration urine collections for twice weekly HD patients, this is likely to be balanced by the incentive for these patients to provide complete urine collections to ensure their dialysis intensity is not increased.

\section{Sample size}

Retrospective studies suggest that decline of RKF may be attenuated in patients who receive twice weekly dialysis compared with thrice weekly, and that this effect occurs early such that a difference in RKF at 6 months is likely to be an optimal time point for the basis of a power analysis. Our initial power analysis, based on our own retrospective data, ${ }^{28}$ indicated an effect size (Cohen's d) of 0.37 calculated from mean and SD of urea clearance slopes 
in the first 6 months after HD initiation between two groups of patients, one initiating HD twice weekly and the other thrice weekly. Based on this, the sample size for the proposed definitive RCT would be 180 (90 each arm). If the definitive study were to be carried out using the same four centres, the available incident HD population would be around 600 annually or 1200 over a proposed 2-year recruitment period. We anticipate that $40 \%$ of these patients will meet the eligibility criteria, that is, 480 patients. To achieve 180 analysable patients at 6 months following randomisation, we will need to recruit $50 \%$ of eligible patients assuming a retention rate of $75 \%$ over 6 months.

This feasibility study will test these assumptions on effect size, the proportion of incident patients who can be prescreened who are eligible to be approached for study consent, the proportion of patients approached for screening who consent, pass formal screening and undergo randomisation (recruitability), and the retention rate during the 6 months after randomisation (retainability). Sample sizes between 24 and 50 have been recommended for feasibility studies. ${ }^{29}{ }^{30}$ Initially, we chose a sample size of 50 but, because of a higher than anticipated recovery of renal function in the first few weeks of recruitment, increased this to 54. A sample of this size will enable us to estimate eligibility, recruitability, screen-failure rate and retainabililty rate to within a $95 \%$ CI $11 \%$ to $14 \%$.

\section{AEs and SAEs}

All AEs will be recorded in an AE log. SAEs will be reported to the CI and sponsors within 24 hours of the research team becoming aware of the event. For the purpose of this study, SAE which result in death, hospitalisation, MACE, infections requiring antibiotic use, episodes of fluid overload needing resetting of dry weight, episodes of hyperkalemia (potassium level $>6.5 \mathrm{mmol} / \mathrm{L}$ ), vascular access events (tunnelled line failures, tunnelled line infections, fistula thrombosis, fistula stenosis, false aneurysm) will be captured.

\section{Data analysis}

The primary outcome is to evaluate the feasibility of conducting an RCT comparing the effect, on RKF decline, of incremental and conventional approaches to HD initiation. The study will be analysed as intention to treat. In order to estimate the study power for a future large-scale RCT, estimates of change in RKF in the first 6 and 12 months after dialysis initiation will be determined.

Change in RKF will be determined using several methods. We will calculate, using linear regression analysis for individual subjects, rate of decline in GFR (mean of urea and creatinine clearance) for individual subjects and compare means of these rates between incremental and conventional HD groups with a t test if normally distributed. This effect size will be important in powering future definitive trials. Using a previously described method we will employ a mixed-effects model to compare rate of decline in GFR between randomisation groups. ${ }^{28}$ As an indicator of RKF, we will compare urine volume data between groups using similar statistical techniques to the above. We will also compare proportions of patients in the two groups who have a residual interdialytic urea clearance $\geq 2$ and $\geq 3 \mathrm{~mL} / \mathrm{min} / 1.73 \mathrm{~m}^{2}$ at 6 months. In addition, we will estimate RKF (GFR) from monthly measured predialysis middle molecule concentrations of $\beta$ trace protein and $\beta 2$-microglobulin converted to an equivalent GFR using the algorithm reported by Wong et al..$^{31}$ We will calculate rate of decline in GFR for individual patients from these middle molecule concentrations and using regression analysis for individual patient data to determine GFR slope and will compare mean slope between incremental HD and standard care groups.

Data from the EQ-5D-5L, PHQ-9, MoCA, IIRS and CFS will be compared between study arms with repeated measures parametric or non-parametric tests as appropriate (repeated measures analysis of variance or Friedman tests). Comparison of MACE, vascular access events (access failure, access intervention, access related infections, fistula stenosis and fistula thrombosis), hyperkalaemic episodes, fluid overload episodes and lower respiratory tract infection episodes will be compared between groups using time-to-event analysis by the Nelson-Aalen approach.

\section{DISCUSSION}

Clinical practice guidelines for HD adequacy, update $2006^{32}$ suggests that reduction of treatment frequency to less than thrice-weekly should only be considered in patients with interdialytic urea clearance $>2 \mathrm{~mL} /$ $\min / 1.73 \mathrm{~m}^{2}$ since urea kinetic modelling simulations have shown that when residual urea clearance is less than this, it is not possible to achieve a weekly Std Kt/V of 2.0 with twice-weekly dialysis regimens. Hence in this study, we have opted for a required interdialytic urea clearance (RKF) of $\geq 3 \mathrm{~mL} / \mathrm{min} / 1.73 \mathrm{~m}^{2} \mathrm{BSA}$ prior to randomisation as an inclusion criterion to provide a safety margin.

There are a large number of observational studies ${ }^{5-18}$ that compare clinical outcomes of patients treated with twice-weekly HD with those on conventional thrice-weekly HD regimens but to date no RCT that compare clinical outcomes of incremental or infrequent HD versus conventional thrice-weekly HD have been published. Though these studies suggest that the rate of decline of RKF is slower using infrequent and incremental HD regimens but prospective, randomised data are not available. Hence it is unclear to what extent the benefits of incremental and infrequent HD are due to patient selection. Similarly, there are no comparative data on quality of life measures or on patient experience in conventional versus incremental HD. Mortality risk and survival outcomes have not been reported to be worse in patients treated with twiceweekly dialysis sessions ${ }^{91316}$ and a large US study found that mortality risk was lower in prevalent patients treated with twice-weekly HD, provided there was adequate RKF. ${ }^{5}$ 
Hence there is a need for a definitive trial of incremental versus conventional dialysis initiation to define the effects on RKF preservation and patient-reported outcome and experience.

The outcome data of this current study will be used to inform the design of such a future definitive study. The proposed feasibility study will test assumptions around the effect size, the eligibility for screening, recruitability and retainability. Deviations from the assumed values will alter the design of the definitive study, for example, number of centres required, eligibility criteria, primary outcome measure, sample size and may indicate that a definitive study is non-viable.

It is likely that the outcomes of a definitive study will be important, not only in defining the potential benefit of incremental HD for patients, but in establishing whether such an approach may allow optimisation of resource use. If dialysis intensity can be reduced for patients with sufficient RKF with patient benefit, this will liberate dialysis resources that may permit other patients with high dialysis requirements to dialyse more frequently.

\section{Author affiliations}

${ }^{1}$ Renal Unit, East and North Hertfordshire NHS Trust, Stevenage, UK

${ }^{2}$ School of Life Sciences, University of Hertfordshire, Hatfield, UK

${ }^{3}$ Renal Unit, Royal Berkshire NHS Foundation Trust, Reading, UK

${ }^{4}$ Renal Unit, University Hospitals of Leicester NHS Trust, Leicester, UK

${ }^{5}$ Renal Unit, Royal Free Hospital, London, UK

Acknowledgements The authors are grateful for the British Kidney Patient Association and British Renal Society Joint Grants Programme for funding this study and for the assistance of our patients in the study design.

Contributors Design of the study and the development of the protocol: EV, RMKK, $\mathrm{KF}$ and SS. Trial setup and running of the study, trial governance, data integrity monitoring: EV and DW. Data analysis: EV, RMKK, KF and DW. Principal investigators: $\mathrm{EV}, \mathrm{JB}, \mathrm{AD}$ and $\mathrm{BA}$. All authors are involved in the steering group of the study and in the analysis and interpretation of the data. All authors read and approved the final manuscript.

Funding This study is funded by the British Kidney Patient Association and British Renal Society Joint Grants Programme (Grant 16-020).

Competing interests None declared.

Patient and public involvement Patients and/or the public were involved in the design, or conduct, or reporting, or dissemination plans of this research. Refer to the Methods section for further details.

Patient consent for publication Not required.

Provenance and peer review Not commissioned; externally peer reviewed.

Open access This is an open access article distributed in accordance with the Creative Commons Attribution Non Commercial (CC BY-NC 4.0) license, which permits others to distribute, remix, adapt, build upon this work non-commercially, and license their derivative works on different terms, provided the original work is properly cited, appropriate credit is given, any changes made indicated, and the use is non-commercial. See: http://creativecommons.org/licenses/by-nc/4.0/.

\section{ORCID iD}

Raja Mohammed Kaja Kamal http://orcid.org/0000-0002-5737-5888

\section{REFERENCES}

1 Kalantar-Zadeh K, Unruh M, Zager PG, et al. Twice-Weekly and incremental hemodialysis treatment for initiation of kidney replacement therapy. Am J Kidney Dis 2014;64:181-6.

2 Davenport A. Will incremental hemodialysis preserve residual function and improve patient survival? Semin Dial 2015;28:16-19.
3 Wong J, Vilar E, Davenport A, et al. Incremental haemodialysis. Nephrol Dial Transplant 2015;30:1639-48.

4 Gilmore J. KDOQI clinical practice guidelines and clinical practice recommendations--2006 updates. Nephrol Nurs J 2006;33:487-8.

5 Hanson JA, Hulbert-Shearon TE, Ojo AO, et al. Prescription of twiceweekly hemodialysis in the USA. Am J Nephrol 1999;19:625-33.

6 Lin Y-F, Huang J-W, Wu M-S, et al. Comparison of residual renal function in patients undergoing twice-weekly versus three-timesweekly haemodialysis. Nephrology 2009;14:59-64.

7 Vilar E, Wellsted D, Chandna SM, et al. Residual renal function improves outcome in incremental haemodialysis despite reduced dialysis dose. Nephrol Dial Transplant 2009;24:2502-10.

8 Supasyndh O, Satirapoj B, Seenamngoen S, et al. Nutritional status of twice and thrice-weekly hemodialysis patients with weekly $\mathrm{Kt} / \mathrm{V}>$ 3.6. J Med Assoc Thai 2009;92:624-31.

9 Lin X, Yan Y, Ni Z, et al. Clinical outcome of twice-weekly hemodialysis patients in Shanghai. Blood Purif 2012;33:66-72.

10 Milagros Fernandez Lucas JLT, Ruiz-Roso G, Diaz M, et al. Incremental hemodialysis schedule in patients with higher residual renal function at the start of dialysis. Advances in Nephrology 2014:6.

11 Zhang M, Wang M, Li H, et al. Association of initial twice-weekly hemodialysis treatment with preservation of residual kidney function in ESRD patients. Am J Nephrol 2014;40:140-50.

12 Bieber B, Qian J, Anand S, et al. Two-times Weekly hemodialysis in China: frequency, associated patient and treatment characteristics and quality of life in the China dialysis outcomes and practice patterns study. Nephrol Dial Transplant 2014;29:1770-7.

13 Elamin S, Abu-Aisha H. Reaching target hemoglobin level and having a functioning arteriovenous fistula significantly improve one year survival in twice Weekly hemodialysis. Arab J Nephrol Transplant 2012;5:81-6.

14 Cheng Y, Tu W, Xiao Q, et al. Risk of cardiovascular disease in patients on thrice-weekly versus twice-weekly hemodialysis. Int $J$ Cardiol 2014;174:780-3.

15 Lei G, Li X, Tu W, et al. Risk of intradialytic hypotension in patients on thrice-weekly versus twice-weekly hemodialysis. Int $J$ Cardiol 2014;174:821-3.

16 Panaput T, Thinkhamrop B, Domrongkitchaiporn S, et al. Dialysis dose and risk factors for death among ESRD patients treated with twice-weekly hemodialysis: a prospective cohort study. Blood Purif 2014;38:253-62.

17 Obi Y, Streja E, Rhee CM, et al. Incremental hemodialysis, residual kidney function, and mortality risk in incident dialysis patients: a cohort study. Am J Kidney Dis 2016;68:256-65.

18 Caria S, Cupisti A, Sau G, et al. The incremental treatment of ESRD: a low-protein diet combined with Weekly hemodialysis may be beneficial for selected patients. BMC Nephrol 2014;15:172.

19 Fernández-Lucas M, Teruel-Briones JL, Gomis-Couto A, et al . Maintaining residual renal function in patients on haemodialysis: 5-year experience using a progressively increasing dialysis regimen. Nefrologia 2012;32:767-76.

20 Devins GM. Using the illness intrusiveness ratings scale to understand health-related quality of life in chronic disease. $J$ Psychosom Res 2010;68:591-602.

21 Herdman M, Gudex C, Lloyd A, et al. Development and preliminary testing of the new five-level version of EQ-5D (EQ-5D-5L). Qual Life Res 2011;20:1727-36.

22 Nasreddine ZS, Phillips NA, Bédirian V, et al. The Montreal cognitive assessment, MoCA: a brief screening tool for mild cognitive impairment. J Am Geriatr Soc 2005;53:695-9.

23 Tiffin-Richards FE, Costa AS, Holschbach B, et al. The Montreal Cognitive Assessment (MoCA) - a sensitive screening instrument for detecting cognitive impairment in chronic hemodialysis patients. PLoS One 2014;9:e106700.

24 Moorhouse P, Rockwood K. Frailty and its quantitative clinical evaluation. J R Coll Physicians Edinb 2012;42:333-40.

25 Alfaadhel TA, Soroka SD, Kiberd BA, et al. Frailty and mortality in dialysis: evaluation of a clinical frailty scale. Clin J Am Soc Nephrol 2015:10:832-40.

26 Gotch FA. The current place of urea kinetic modelling with respect to different dialysis modalities. Nephrol Dial Transplant 1998;13:10-14.

27 Casino FG, Lopez T. The equivalent renal urea clearance: a new parameter to assess dialysis dose. Nephrol Dial Transplant 1996;11:1574-81.

28 Kaja Kamal RM, Farrington K, Busby AD, et al. Initiating haemodialysis twice-weekly as part of an incremental programme may protect residual kidney function. Nephrol Dial Transplant 2019;34:1017-25.

29 Julious SA. Sample size of 12 per group rule of thumb for a pilot study. Pharm Stat 2005;4:287-91. 
$30 \operatorname{Sim} \mathrm{J}$, Lewis M. The size of a pilot study for a clinical trial should be calculated in relation to considerations of precision and efficiency. $J$ Clin Epidemiol 2012;65:301-8.

31 Wong J, Sridharan S, Berdeprado J, et al. Predicting residual kidney function in hemodialysis patients using serum $\beta$-trace protein and ß2-microglobulin. Kidney Int 2016;89:1090-8.
32 Hemodialysis Adequacy 2006 Work Group. Clinical practice guidelines for hemodialysis adequacy, update 2006. Am J Kidney Dis 2006;48:S2-90. 